\title{
Validation and comparison of Diagnosis of TIA (DOT) score as a new clinical diagnostic tool for patients with a clinical diagnosis of transient ischemic attack in China
}

Junliang Yuan

Beijing Chaoyang Hospital

Zejin Jia

Beijing Chaoyang Hospital

Yangguang Song

Beijing Chaoyang Hospital

Wenli Hu ( $\sim$ huwenli@sina.com )

Beijing Chaoyang Hospital Affiliated to Capital Medical University https://orcid.org/0000-0002-94439203

Research article

Keywords: transient ischemic attack, diagnosis of TIA score, ABCD3-I, Dawson

Posted Date: April 4th, 2019

DOI: https://doi.org/10.21203/rs.2.1817/v1

License: (9) This work is licensed under a Creative Commons Attribution 4.0 International License.

Read Full License 


\section{Abstract}

Background: Recently, [the diagnosis of transient ischemic attack (TIA), DOT) score was considered to be a new tool for non-specialists to diagnose TIA more accurately with the sensitivity and specificity were $89 \%$ and $76 \%$. However, the DOT score has not yet been validated externally in patients with TIA in China. Methods: We prospectively enrolled consecutive 500 patients with transient neurological symptoms, who were admitted to our hospital and underwent magnetic resonance imaging (MRI) during hospitalization between Jan 2016 and Dec 2018. Patients with transient neurological symptoms were divided into two subgroups: TIA mimic group ( $N=140,28 \%)$ and definite cerebrovascular events group (including tissuebased TIA $(N=252,50.4 \%)$ and minor stroke $(N=108,21.6 \%))$. The demographic data, clinical characteristics, laboratory findings and scores of ABCD2, ABCD3-I, Dawson and DOT were compared between the two groups. Results: A total of 500 patients with transient neurological symptoms (mean age, 61.1 \pm 12.8$)$ were enrolled and $70 \%(\mathrm{~N}=350)$ were male. Comparing with TIA mimic groups, patients with definite cerebrovascular events group were correlated with higher diastolic blood pressure, higher proportion of motor weakness and speech abnormalities, the higher levels of uric acid and homocysteine, and the scores of ABCD2, ABCD3-I, Dawson, and DOT. The logistic regression analysis showed diastolic blood pressure (OR 1.026, Cl 1.006-1.046, P=0.011), ABCD3-I (OR 1.219, Cl 1.032-1.440, P=0.020) and DOT (OR 1.462, Cl 1.305-1.637, $\mathrm{P}=0.001$ ) were independently predicted the occurrence of definite cerebrovascular events. The score of DOT showed the greatest area under the ROC curve (AUC=0.728), with a sensitivity of $70.3 \%$ and specificity of $62.9 \%$, respectively. The optimal cut point for DOT was 0.455 using the Youden Index. Conclusion: Our findings showed DOT score performed relatively good calibration and discrimination. As a novel tool of TIA, further validations are needed externally in patients with TIA with multiple centers in large samples in China.

\section{Background}

Stroke is ranked as the second leading cause of death worldwide with an annual mortality rate of about 5.5 million [1, 2], and the first one in China. It has been reported that $20 \%$ of stroke is preceded by an episode of transient ischemic attack (TIA). Patients with TIA have the highest risk of early/recurrent stroke or other cardiovascular events [3-5]. Thus, it is worthwhile to develop reliable risk scores to prevent stroke in an early stage, and to apply standard interventions before a stroke occurs. The ABCD2 scale (range 0 to 7) was firstly developed to provide a structured way to stratify the early risk of stroke after TIA $[6,7]$, with higher scores indicating a higher risk of stroke. Later, ABCD2-I score (range 0 to 10) [8], ABCD3 (range 0 to 9) [9], ABCD3-I score (range 0 to 13) [10,11] have been also widely used to evaluate the risk factors of stroke, however, a third of mimics were found to have ABCD2 scores $\geq 4$ [12].

TIA represents a medical emergency, and the focus should be on differentiating TIA from stroke and common mimics [13]. As for diagnostic algorithms for TIA, there have been only two tools developed. The first one was Dawson score, which was a clinical scoring tool developed in a specialist setting. However, it was limited utility in a primary care setting and not including the retinal and posterior circulation cerebrovascular events [14]. The second one was the Diagnosis of TIA (DOT) score, which was 
considered to be a new tool for non-specialists to make the diagnosis of TIA with greater accuracy with the sensitivity and specificity were $89 \%$ and $76 \%$, respectively. The area under the curve (AUC) of DOT score was 0.89 , however, the Dawson score was 0.77 [15]. However, this new tool of DOT has not yet been externally validated in other populations before widely utilized. Thus, the aim of the present study is to investigate the validation and comparison of DOT score as a new clinical diagnostic tool for a clinical diagnosis of TIA in China.

\section{Methods}

\section{Patients}

Our study was a retrospective, observational study in the department of Neurology, Beijing Chaoyang Hospital, Capital Medical University. We retrospectively enrolled consecutive 500 patients with transient neurological symptoms between Jan 2016 and Dec 2018 and they all underwent magnetic resonance imaging (MRI) scans [including diffusion weighted imaging (DWI)] within 7 days after the onset. Patients with transient neurological symptoms were classified to be TIA, minor stroke or mimic [15]. TIA is classically defined to be the presence of focal neurological symptoms due to a vascular etiology lasting less than 24 hours, irrespective of imaging findings [16]. The tissue-based definition of TIA was proposed to classify patients only if the symptoms fit the clinical syndrome and no acute ischemic lesion identified by MRI [17]. Patients with transient neurological symptoms were divided into two subgroups: TIA mimic group and definite cerebrovascular events group (including tissue-based TIA and minor stroke). The demographic data, clinical features, and the scores of TIA assessment were analyzed. Our work was approved by the Ethics Committee of Beijing Chaoyang Hospital, Capital Medical University. Written informed consent was obtained from all participants.

\section{Clinical Variables}

We retrospectively obtained the following clinical data: age, gender; vascular risk factors such as hypertension, diabetes mellitus, hyperlipidemia, stroke, coronary heart disease, atrial fibrillation and current smoking. The laboratory blood tests were obtained from medical records, including the counts of red blood cell, white blood cell, platelet, hemoglobin, fibrinogen, fasting blood glucose, hemoglobin A1c, glycated albumin, uric acid, homocysteine, total cholesterol, low-density lipoprotein, high-density lipoprotein, triglyceride, C-reactive protein, albumin, pre-albumin, VitB12, folic acid, 25-hydroxy vitamin D, brain natriuretic peptide. The score s of ABCD2, ABCD3-I, Dawson and DOT were also calculated. The presenting symptoms of TIA included motor weakness, sensory disturbance, speech abnormalities (dysarthria/aphasia), loss of consciousness, ataxia, diplopia, hemianopia. The durations of TIA were classified into 3 categories: $<10 \mathrm{~min}, 10-59 \mathrm{~min}$, or more than 1 hour.

\section{Statistical Analysis}

The data were described using the mean and standard deviation values for continuous variables, the median and interquartile range values for categorical variables, and absolute numbers and percentages for nominal and categorical variables, and we compared the groups using the nonparametric Mann- 
Whitney $\mathrm{U}$ test. We performed a chi-square test to determine the correlation between categorical variables and a t test between continuous variables. According to Dutta D study, the dependent variable was "definite cerebrovascular events", which included tissue-based TIA and minor stroke [15]. The backward multivariate regression analysis was performed to find predictors of cerebrovascular events. The calibration of the models was tested by calibration plots and the Hosmer-Lemeshaw statistic, and the discrimination was tested by Receiver Operating Characteristic (ROC) curves and AUC. We used the Statistical Package for Social Sciences (SPSS) version 16.0 (SPSS Inc., Chicago, IL, USA) for data analysis. A $P$ value less than 0.05 was considered statistically significant.

\section{Results}

We enrolled a total of 500 patients with transient neurological symptoms, and the mean age was $61.1 \pm 12.8$ years, and $70 \%(\mathrm{~N}=350)$ were male. Among the patients with TIA symptoms, we found that TIA mimic $(N=140,28 \%)$ and cerebrovascular events [including tissue-based TIA $(N=252,50.4 \%)$ and minor stroke $(\mathrm{N}=108,21.6 \%)]$.

Table 1 showed the demographics, baseline clinical characteristics, vascular risk factors, TIA symptoms and the duration of TIA. The laboratory findings and the scores of TIA were presented in Table 2. We found there were significant differences in diastolic blood pressure, the proportion of motor weakness and speech abnormalities, the levels of uric acid and homocysteine, and the scores of ABCD2, ABCD3-I, Dawson and DOT between the two groups $(P<0.05)$.

In backwards multivariable logistic regression analysis in Table 3, we found the diastolic blood pressure (OR 1.026, Cl1.006-1.046, $P=0.011)$, ABCD3-I (OR 1.219, C/1.032-1.440, $P=0.020)$ and DOT (OR 1.462, Cl $1.305-1.637, P=0.001)$ were independently predicted the occurrence of cerebrovascular events.

As for the calibration and discrimination, predicted and observed diagnoses were plotted to test calibration and discrimination was tested by the AUC with $95 \% \mathrm{Cl}$. Figure 1 showed the calibration plots (predicted vs observed results) of the final model in our study. The $P$ value was 0.541 for the HosmerLemeshow test of goodness of fit in our dta. From Table 4 and Figure 2, we found that the area under the ROC curve $(95 \% \mathrm{Cl})$ of the scores of ABCD2, ABCD3-I, Dawson and DOT for cerebrovascular events prediction were 0.606 (95\% Cl, 0.551-0.660), 0.621 (95\% Cl, 0.567-0.674), 0.681 (95\% Cl, 0.629-0.732), 0.728 (95\% Cl, 0.680-0.776), respectively. The score of DOT showed the greatest area under the ROC curve, with a sensitivity of $70.3 \%$ and specificity of $62.9 \%$, respectively. The optimal cut point for DOT score was 0.455 using the Youden Index.

\section{Discussion}

In our present study, we found that diastolic blood pressure, the score of ABCD3-I and DOT were independently associated with the occurrence of definite cerebrovascular events. Besides, DOT score also performed relatively good calibration and discrimination, with the greatest area under the ROC curve. 
The definition of TIA has been changing with the development of advanced techniques of neuroimaging. TIA was classically defined as a focal cerebral ischemic event with symptoms lasting $<24$ hours. Due to the rapid development of neuroimaging, up to one-third of patients with TIA may have radiological evidence of acute infarction $[18,19]$. The frequency of positive DWI findings varied from 9 to $67 \%$ among different cohorts of patients with TIA $[8,20,21]$. Therefore, the definition of TIA is moving from "timebased TIA" to "tissue-based TIA" as "a transient episode of neurological dysfunction caused by focal brain, spinal cord, or retinal ischemia, without acute infarction" [17]. Changing the definition of TIA from time to a tissue basis could produce a landmark of the development and progress of stroke.

TIA requires urgent investigation and it has been poorly managed in many sites. The term of 'transient neurological symptoms' is used to describe the broad spectrum of symptoms following TIA or TIA mimics. Unilateral weakness and speech disturbances are the most common manifestations of TIA [22]. It was reported unilateral weakness and speech disturbance were found in approximately $31 \%-54 \%$ and $25 \%-42 \%$ of TIA, respectively [18]. There is no gold standard clinical tool used to diagnose a TIA or stroke based on symptomology, however, the diagnosis of TIA needs the assessment of symptoms and adequate evaluation by a stroke specialist. However, accurate identification of stroke and TIA patients is quite difficult. It was reported up to $50 \%$ to $60 \%$ of TIA patients were diagnosed to be noncerebrovascular mimics by non-specialists [23]. Another report showed that $35 \%$ of referrals to TIA clinics were nonvascular mimics [24]. As a result, it is vital and valuable that the differential diagnosis of transient neurological symptoms including both patients with TIA and mimics (e.g. migraine or seizure) $[25,26]$.

In spite that MRI may be a very useful tool and certainly reduces the rate of false-negative diagnoses, it still cannot replace clinical assessment, especially for those patients with MRI-negative. As a result, the clinical evaluation could be quite important to stratify different risk factors of TIA patients. Risk stratification instruments such as the scores of $A B C D$ scores series (such as $A B C D, A B C D 2, A B C D 3$, $A B C D 3-I)$ could be available but were developed on populations with confirmed TIA. The ABCD2 scores which were recorded by the referring clinician, showed very poor discrimination for the diagnosis of TIA [27]. To date, the ABCD3-I scores has been considered to be the best validated and predominantly used risk evaluators in TIA patients $[28,29]$. However, due to the fact that MRI is not available in all healthcare settings and medical emergency, ABCD3-I scores could also have some limits in our clinical practice. A TIA diagnostic tool could be utilized to improve TIA clinic triage by differentiating some TIA mimics from urgent TIA pathways. To date, there have been only two diagnostic algorithms for the early diagnosis of TIA. The clinical scoring system of Dawson was proved to facilitate accurate detection of TIA. However, it was limited to use in a primary care setting and not involving the retinal and posterior circulation cerebrovascular events [14]. The second one was the DOT score, which was considered to be a new tool for non-specialists to make the diagnosis of TIA with greater accuracy [15]. However, this new tool of DOT has not yet been externally validated in other populations out of the United Kingdom. Our findings indicated that DOT score performed relatively good calibration and discrimination, with a sensitivity of $70.3 \%$ and specificity of $62.9 \%$, respectively. We also found that DOT performed the greatest area under the ROC curve, and ABCD3-I ranked the second greatest area. According to Dutta D study in 2016, the AUC for DOT was 0.89 and Dawson score was 0.77 , while ABCD2 was 0.64 . In spite that our 
data were not as perfect as Dutta D study, however, our findings were consistent with it. It was reported that the AUC of ABCD2 score and ABCD3-I score was 0.50 and 0.58 in South Korea population [30]. In the population of Spain, the area under the ROC curve of $A B C D 2$ and $A B C D 3-I$ for ischemic events prediction were 0.49 and 0.77 [31]. Recently, the ABCD3-I score performed equally in TIA patients in tissue- as well as time-based definition and the same for minor stroke patients [22]. Our study was also in line with the above studies.

Our study had some limitations which should be raised. Firstly, this study was designed as a retrospective study from a single center, which was a great disadvantage. Studies with a larger numbers from multiple centers in China are needed urgently to further confirm our findings. Secondly, we did not perform followups to assess of the risk factors of the recurrence of TIA or stroke, and not get the time from symptom onset to DWI examination (time-to-DWI). The above issues may be also great importance for the assessment and management of TIA. Thirdly, our study also lacked of stratification such as low, median, high subgroup according to ABCD2/ABCD3-I scores; and we did not perform the etiological classification of stroke/TIA, according to the Trial of Org 10172 in Acute Stroke Treatment. Fourthly, we did not find blood or imaging biomarkers available to reliably distinguish TIA from TIA-mimics [32]. Despite these limitations, as far as we know, the present study is the largest ever-reported study to identify the clinical predictors for the diagnosis of TIA. Besides, for the first time, we have externally validated the DOT score as for a new tool of TIA in patients with TIA in China. Thirdly, we further conformed that the score of DOT could encompass the entire spectrum of TIA/stroke, which could be used as a mobile app and web based calculator with more accurate to assess.

\section{Conclusion}

In summary, in patients with TIA symptoms, our findings showed that the occurrence of cerebrovascular events was independently associated with the diastolic blood pressure, the scores of ABCD3-I and DOT. We have also externally validated the DOT in patients with TIA, and it was proved to be accurate as a diagnostic tool for TIA in China. The relative merits and clinical utility of DOT score will warrant further prospective study in multiple centers with large samples.

\section{Abbreviations}

TIA: transient ischemic attack; MRI: magnetic resonance imaging; DOT: the diagnosis of transient ischemic attack; AUC: under the curve; DWl: diffusion weighted imaging; ROC: receiver operating characteristic.

\section{Declarations}

\section{Acknowledgements}

We thank Dr. Yicheng Xu, Weixue Wang, Shang Wang, Zihan Yan, Yu Zhang for their great help for the data collection and analysis. 


\section{Funding}

This work was supported by the National Natural Science Foundation of China (81301016) and the Beijing Municipal Administration of Hospitals Incubating Program (PX2019009). The two findings help us data collection, analysis, and interpretation of data.

\section{Availability of Data and Materials}

The datasets will be available on request, provided that the Ethics Committee gives approval.

\section{Authors' contributions}

JLY, ZJJ, YGS examined, evaluated the patient. JLY drafted and revised the manuscript. WLH participated in the design of the case-report and helped to draft the manuscript. All authors read and approved the final manuscript.

\section{Ethics Approval and Consent to Participate}

The study was approved by the Institutional Ethical Committee of Beijing Chaoyang Hospital, Capital Medical University. Written informed consent was obtained from all subjects before study inclusion

\section{Consent for publication}

Written informed consent was obtained from all the subjects.

\section{Competing interests}

The authors declare that they have no competing interests.

\section{References}

1. Donkor ES. Stroke in the 21(st) Century: A Snapshot of the Burden, Epidemiology, and Quality of Life. Stroke research and treatment. 2018;2018:3238165.

2. Katan M, Luft A. Global Burden of Stroke. Seminars in neurology. 2018;38 2:208-11.

3. Giles MF, Rothwell PM. Risk of stroke early after transient ischaemic attack: a systematic review and meta-analysis. The Lancet Neurology. 2007;6 12:1063-72.

4. Coutts SB, Modi J, Patel SK, Demchuk AM, Goyal M, Hill MD. CT/CT angiography and MRI findings predict recurrent stroke after transient ischemic attack and minor stroke: results of the prospective CATCH study. Stroke. 2012;43 4:1013-7.

5. Hao Q, Tampi M, O'Donnell M, Foroutan F, Siemieniuk RA, Guyatt G. Clopidogrel plus aspirin versus aspirin alone for acute minor ischaemic stroke or high risk transient ischaemic attack: systematic review and meta-analysis. BMJ (Clinical research ed). 2018;363:k5108. 
6. Johnston SC, Rothwell PM, Nguyen-Huynh MN, Giles MF, Elkins JS, Bernstein AL, et al. Validation and refinement of scores to predict very early stroke risk after transient ischaemic attack. Lancet (London, England). 2007;369 9558:283-92.

7. Giles MF, Rothwell PM. Systematic review and pooled analysis of published and unpublished validations of the ABCD and ABCD2 transient ischemic attack risk scores. Stroke. 2010;41 4:667-73.

8. Giles MF, Albers GW, Amarenco P, Arsava MM, Asimos A, Ay H, et al. Addition of brain infarction to the $A B C D 2$ Score (ABCD2I): a collaborative analysis of unpublished data on 4574 patients. Stroke. 2010;41 9:1907-13.

9. Johansson E, Bjellerup J, Wester P. Prediction of recurrent stroke with ABCD2 and ABCD3 scores in patients with symptomatic 50-99\% carotid stenosis. BMC neurology. 2014;14:223.

10. Dai Q, Sun W, Xiong Y, Hankey GJ, Xiao L, Zhu W, et al. From clinical to tissue-based dual TIA: Validation and refinement of ABCD3-I score. Neurology. 2015;84 14:1426-32.

11. Kiyohara T, Kamouchi M, Kumai Y, Ninomiya T, Hata J, Yoshimura S, et al. ABCD3 and ABCD3-I scores are superior to ABCD2 score in the prediction of short- and long-term risks of stroke after transient ischemic attack. Stroke. 2014;45 2:418-25.

12. Wardlaw JM, Brazzelli M, Chappell FM, Miranda H, Shuler K, Sandercock PA, et al. ABCD2 score and secondary stroke prevention: meta-analysis and effect per 1,000 patients triaged. Neurology. $2015 ; 85$ 4:373-80.

13. Siket MS, Edlow J. Transient ischemic attack: an evidence-based update. Emergency medicine practice. 2013;15 1:1-26.

14. Dawson J, Lamb KE, Quinn TJ, Lees KR, Horvers M, Verrijth MJ, et al. A recognition tool for transient ischaemic attack. Qjm. 2009;102 1:43-9.

15. Dutta D. Diagnosis of TIA (DOT) score-design and validation of a new clinical diagnostic tool for transient ischaemic attack. BMC neurology. 2016;16:20.

16. Special report from the National Institute of Neurological Disorders and Stroke. Classification of cerebrovascular diseases III. Stroke. 1990;21 4:637-76.

17. Easton JD, Saver JL, Albers GW, Alberts MJ, Chaturvedi S, Feldmann E, et al. Definition and evaluation of transient ischemic attack: a scientific statement for healthcare professionals from the American Heart Association/American Stroke Association Stroke Council; Council on Cardiovascular Surgery and Anesthesia; Council on Cardiovascular Radiology and Intervention; Council on Cardiovascular Nursing; and the Interdisciplinary Council on Peripheral Vascular Disease. The American Academy of Neurology affirms the value of this statement as an educational tool for neurologists. Stroke. 2009;40 6:2276-93. 
18. Giles MF, Albers GW, Amarenco P, Arsava EM, Asimos AW, Ay H, et al. Early stroke risk and ABCD2 score performance in tissue- vs time-defined TIA: a multicenter study. Neurology. 2011;77 13:1222-8.

19. Wardlaw J, Brazzelli M, Miranda H, Chappell F, McNamee P, Scotland G, et al. An assessment of the cost-effectiveness of magnetic resonance, including diffusion-weighted imaging, in patients with transient ischaemic attack and minor stroke: a systematic review, meta-analysis and economic evaluation. Health technology assessment (Winchester, England). 2014;18 27:1-368.

20. Crisostomo RA, Garcia MM, Tong DC. Detection of diffusion-weighted MRI abnormalities in patients with transient ischemic attack: correlation with clinical characteristics. Stroke. 2003;34 4:932-7.

21. Redgrave JN, Coutts SB, Schulz UG, Briley D, Rothwell PM. Systematic review of associations between the presence of acute ischemic lesions on diffusion-weighted imaging and clinical predictors of early stroke risk after transient ischemic attack. Stroke. 2007;38 5:1482-8.

22. Mayer L, Ferrari J, Krebs S, Boehme C, Toell T, Matosevic B, et al. ABCD3-I score and the risk of early or 3-month stroke recurrence in tissue- and time-based definitions of TIA and minor stroke. Journal of neurology. 2018;265 3:530-4.

23. Dutta D, Bowen E, Foy C. Four-year follow-up of transient ischemic attacks, strokes, and mimics: a retrospective transient ischemic attack clinic cohort study. Stroke. 2015;46 5:1227-32.

24. Giles MF, Rothwell PM. Substantial underestimation of the need for outpatient services for TIA and minor stroke. Age and ageing. 2007;36 6:676-80.

25. Sheehan OC, Merwick A, Kelly LA, Hannon N, Marnane M, Kyne L, et al. Diagnostic usefulness of the $A B C D 2$ score to distinguish transient ischemic attack and minor ischemic stroke from noncerebrovascular events: the North Dublin TIA Study. Stroke. 2009;40 11:3449-54.

26. Brazzelli M, Chappell FM, Miranda H, Shuler K, Dennis M, Sandercock PA, et al. Diffusion-weighted imaging and diagnosis of transient ischemic attack. Ann Neurol. 2014;75 1:67-76.

27. Quinn TJ, Cameron AC, Dawson J, Lees KR, Walters MR. ABCD2 scores and prediction of noncerebrovascular diagnoses in an outpatient population: a case-control study. Stroke. 2009;40 3:74953.

28. Song B, Fang H, Zhao L, Gao Y, Tan S, Lu J, et al. Validation of the ABCD3-I score to predict stroke risk after transient ischemic attack. Stroke. 2013;44 5:1244-8.

29. Merwick A, Albers GW, Amarenco P, Arsava EM, Ay H, Calvet D, et al. Addition of brain and carotid imaging to the $A B C D(2)$ score to identify patients at early risk of stroke after transient ischaemic attack: a multicentre observational study. The Lancet Neurology. 2010;9 11:1060-9. 
30. Nah HW, Kwon SU, Kang DW, Lee DH, Kim JS. Diagnostic and prognostic value of multimodal MRI in transient ischemic attack. Int J Stroke. 2014;9 7:895-901.

31. Purroy F, Jimenez-Caballero PE, Mauri-Capdevila G, Torres MJ, Gorospe A, Ramirez Moreno JM, et al. Predictive value of brain and vascular imaging including intracranial vessels in transient ischaemic attack patients: external validation of the ABCD3-I score. European journal of neurology. 2013;20 7:108893.

32. Fitzpatrick T, Gocan S, Wang CQ, Hamel C, Bourgoin A, Dowlatshahi D, et al. How do neurologists diagnose transient ischemic attack: A systematic review. Int J Stroke. 2018:1747493018816430.

\section{Tables}

Table 1 The baseline demographics, clinical characteristics between the two groups 
Demographics

\begin{tabular}{|c|c|c|c|}
\hline $\operatorname{Age}(Y)$ & $60.1 \pm 12$ & $61.5 \pm 13.1$ & 0.273 \\
\hline Sex(Male, \%) & $90(64.3 \%)$ & $260(72.2 \%)$ & 0.082 \\
\hline Systolic blood pressure(mmHg) & $143.6 \pm 20.4$ & $146.9 \pm 21.2$ & 0.117 \\
\hline Diastolic blood pressure(mmHg) & $79.8 \pm 11.6$ & $83.5 \pm 13.6$ & $0.003^{*}$ \\
\hline \multicolumn{4}{|l|}{ Vascular Risk factors } \\
\hline Hypertension & $78(55.7 \%)$ & $229(63.6 \%)$ & 0.103 \\
\hline Diabetes mellitus & $50(31.2 \%)$ & $110(26.5 \%)$ & 0.267 \\
\hline Coronary disease & $8(5.7 \%)$ & $28(7.8 \%)$ & 0.423 \\
\hline Prior stroke & $22(15.7 \%)$ & $71(19.7 \%)$ & 0.301 \\
\hline Hypercholesterolemia & 104(74.3\%) & $255(70.8 \%)$ & 0.441 \\
\hline Atrial fibrillation & $2(1.4 \%)$ & $12(3.3 \%)$ & 0.246 \\
\hline Peripheral arterial disease & $5(3.6 \%)$ & $5(1.4 \%)$ & 0.118 \\
\hline Smoking & $59(42.1 \%)$ & $184(51.1 \%)$ & 0.072 \\
\hline \multicolumn{4}{|l|}{ Clinical features } \\
\hline Motor weakness & $48(34.3 \%)$ & $231(64.2 \%)$ & $0.001^{*}$ \\
\hline Sensory disturbance & $31(22.1 \%)$ & $105(29.2 \%)$ & 0.113 \\
\hline Speech abnormalities & $36(25.7 \%)$ & $145(40.3 \%)$ & $0.002^{*}$ \\
\hline Ataxia & $7(5.0 \%)$ & $19(5.3 \%)$ & 0.900 \\
\hline Diplopia & $9(6.4 \%)$ & $19(5.3 \%)$ & 0.615 \\
\hline Loss of consciousness & $16(11.4 \%)$ & $25(6.9 \%)$ & 0.101 \\
\hline Hemianopia & $2(1.4 \%)$ & $14(3.9 \%)$ & 0.160 \\
\hline Duration of TIA & & & 0.846 \\
\hline$<10 \min$ & $60(45.5 \%)$ & $150(47.6 \%)$ & \\
\hline $10-59 \min$ & $50(37.9 \%)$ & $142(40.3 \%)$ & \\
\hline $1 \mathrm{~h}$ & $22(16.7 \%)$ & $60(17 \%)$ & \\
\hline
\end{tabular}

Data were presented as median with interquartile range in parentheses or number with percentage in parentheses. ${ }^{*} P<0.05$; 
Table 2 The laboratory findings and the risk scores of TIA between the two groups 
Variables

White blood cell (109/L)

Red blood cell (1012/L)

Hemoglobin $(\mathrm{g} / \mathrm{L})$

Platelet (109/L)

Albumin $(\mathrm{g} / \mathrm{L})$

Pre-albumin $(\mathrm{g} / \mathrm{L})$

Total cholesterol $(\mathrm{mmol} / \mathrm{L})$

High-density lipoprotein (mmol/L) $\quad 1.1 \pm 0.3$

$2.8 \pm 0.8$

$1.8 \pm 1.5$

$312.2 \pm 89.1$

$15.1 \pm 7$

$572.6 \pm 559$

$6.7 \pm 5.1$

$14.5 \pm 4.8$

$2.4 \pm 2.8$

$46.4 \pm 38.7$

$267.3 \pm 66.6$

$6.4 \pm 2.3$

$6.3 \pm 1.1$

$13.7 \pm 3.6$

$3(2-4)$

5(3-6)

$6.5 \pm 1.3$

$-0.04(-2.1 \otimes 0.92)$
$1.25(0.31 \otimes 3.47)$

0.636

0.999

0.989

0.798

0.363

0.285

0.239

0.449

0.07

0.296

$0.004^{*}$

$0.007 *$

$478.5 \pm 451.8$

0.072

$6.1 \pm 4.8$

0.251

0.752

0.152

0.111

0.468

0.209

0.880

0.398

0.001 *

$0.001^{*}$

$0.001^{*}$

$0.001^{*}$ 
Values represent number (percent) for categorical variables and mean \pm SD or median (interquartile range) for continuous variables. ${ }^{*} P<0.05$;

Abbreviations: DOT, diagnosis of TIA.

Table 3 Multivariate logistic regression as predictors of definite cerebrovascular events

\begin{tabular}{lllll} 
Predictors & $P$ & $O R$ & $95 \%$ C/for $O R$ & \\
\cline { 1 - 4 } Diastolic blood pressure & $0.011 *$ & 1.026 & 1.006 & 1.046 \\
\cline { 1 - 4 } ABCD3-I & $0.020 *$ & 1.219 & 1.032 & 1.44 \\
\hline DOT & $0.001 *$ & 1.462 & 1.305 & 1.637
\end{tabular}

$\star P<0.05$

Table 4 The area under the ROC curve $(95 \% \mathrm{Cl})$ of the scores of TIA

\begin{tabular}{lllll} 
Variable(s) & Area Under the Curve & $P$ & $95 \% \mathrm{Cl}$ & \\
\cline { 1 - 2 } & & & & \\
ABCD2 & 0.606 & $0.001 *$ & 0.551 & 0.660 \\
\hline ABCD3I & 0.621 & $0.001 *$ & 0.567 & 0.674 \\
\hline Dawson & 0.681 & $0.001 *$ & 0.629 & 0.732 \\
\hline DOT & 0.728 & $0.001 *$ & 0.680 & 0.776
\end{tabular}

$* P<0.05$

Figures 


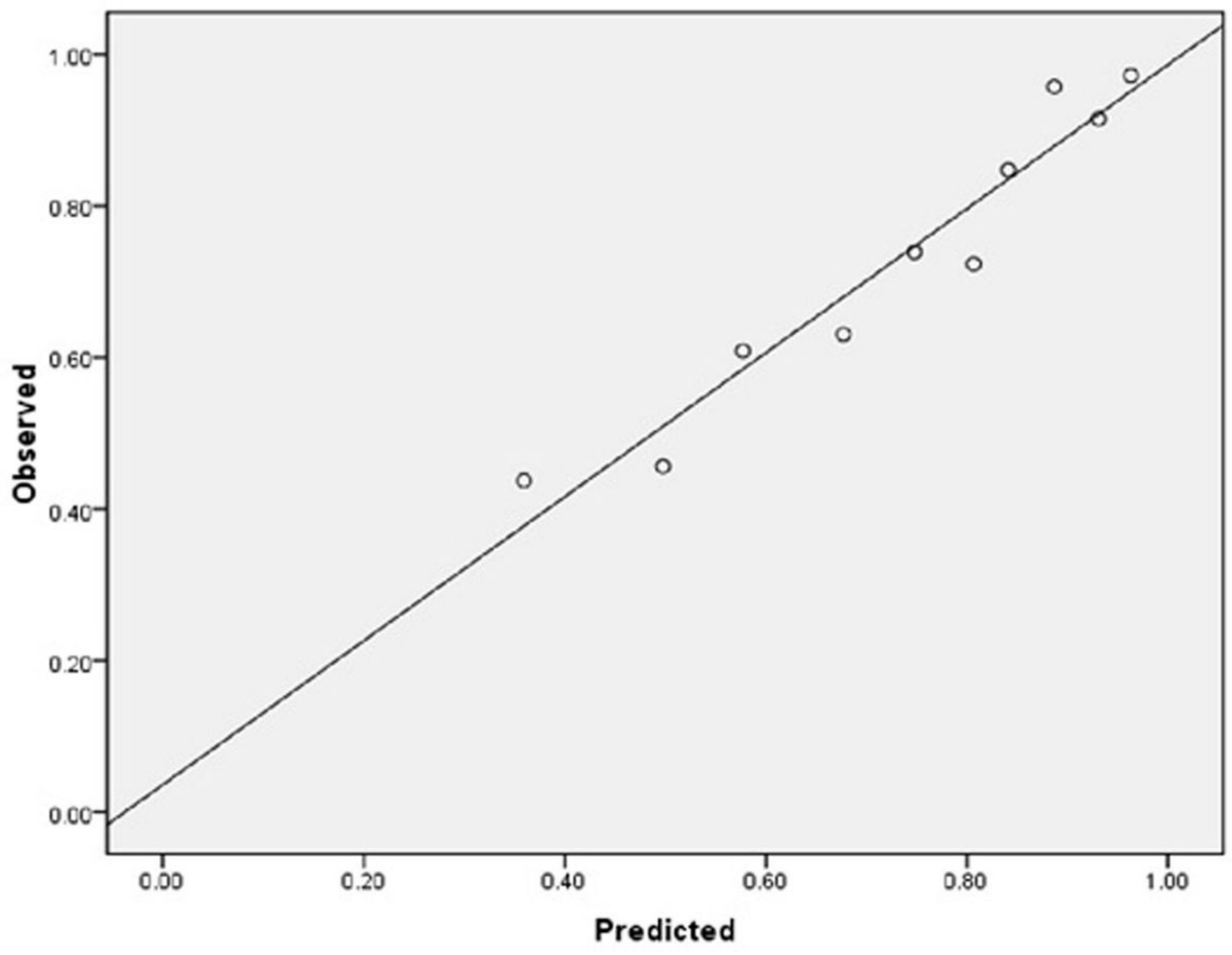

Figure 1

The calibration plots (predicted vs observed results) of the final model in our study 


\section{ROC Curve}

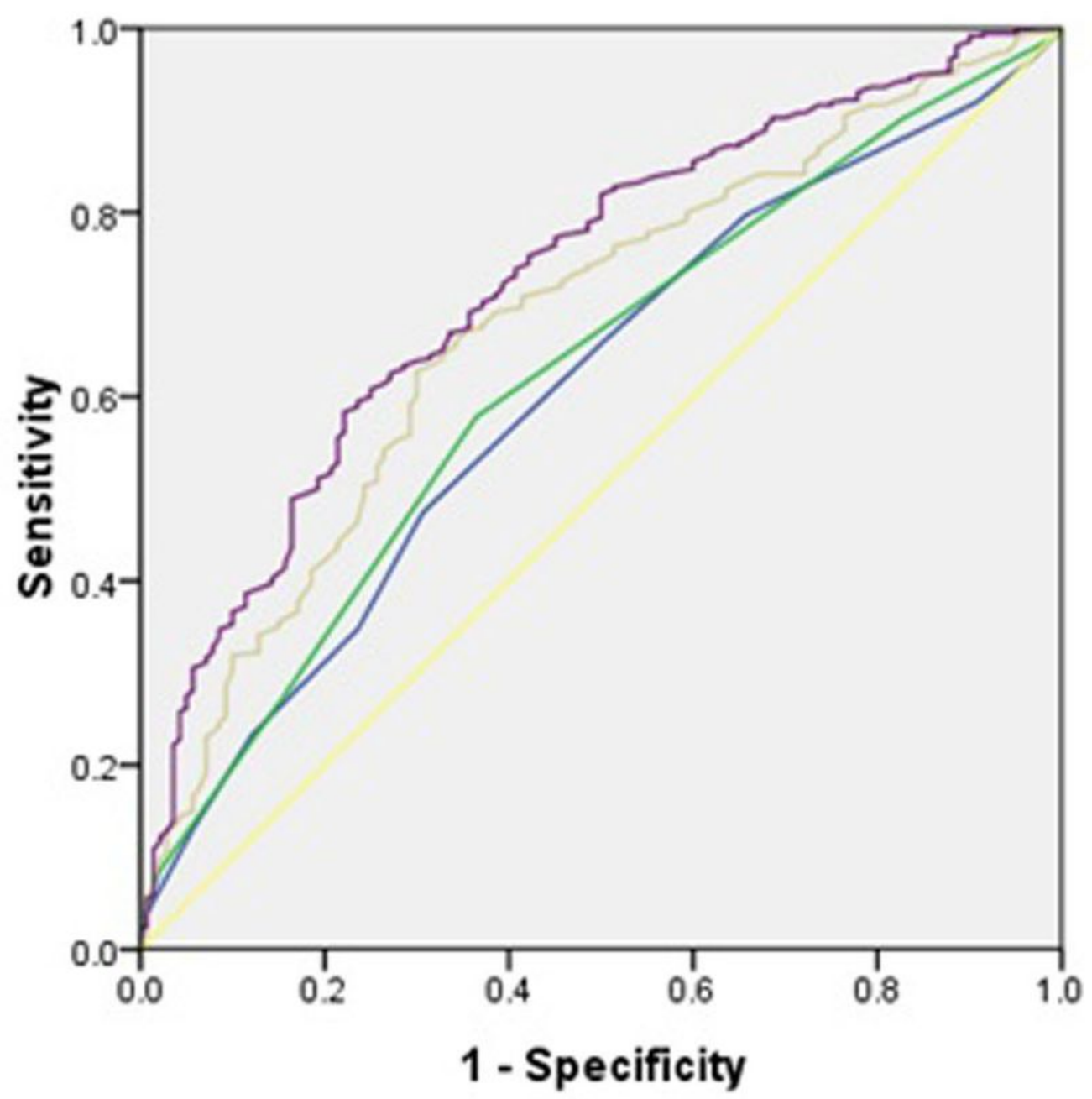

Source of the Curve

$-\mathrm{ABCD} 2$

$-\mathrm{ABCD} 31$

Dawson

- DOT

Reference Line

Figure 2

ROC curves for predictive value of the scores of $A B C D 2, A B C D 3-I$, Dawson and DOT 